\title{
Instability Characteristics of a Free-Free Multi-Stepped Timoshenko Beam with Concentrated Masses Subjected to Follower Force
}

\author{
By Amir H. Esbati, ${ }^{1)}$ Saied Irani ${ }^{1)}$ and Hamid MoosaZAdeH ${ }^{2)}$ \\ ${ }^{1)}$ Faculty of Aerospace Engineering, K. N. Toosi University of Technology, Tehran, Iran \\ ${ }^{2)}$ Department of Aerospace, Tarbiat Modares University, Tehran, Iran
}

(Received May 13th, 2010)

\begin{abstract}
This paper presents dynamic and static instability characteristics of a free-free multi-stepped Timoshenko beam subjected to a follower force, where each step has a point mass with transverse and rotary inertia. A finite element code is developed to model two-dimensional multi-stepped Timoshenko beams using the extended Hamilton's principle. The effects of each section parameters on the type of instability and critical follower force have been studied for different models of multi-stepped beams. It is concluded that the assumption of a uniform beam to study most aerospace structures such as launch vehicles could lead to an unrealistic instability analysis, where any small change in the characteristics parameters for each step could change the instability behavior significantly. Furthermore, this paper introduces a new parametric approach concept for analyzing the characteristics effects of a multi-step aerospace structure.
\end{abstract}

Key Words: $\quad$ Multi-Stepped Timoshenko Beam, Follower Force, Finite Element, Static and Dynamic Instability

\section{Nomenclature}

$A_{i}:$ cross-sectional area

$A(\tilde{\xi})$ : Timoshenko shape function

$A D i$ : diameter ratio of $i$ th step

$B(\tilde{\xi})$ : Timoshenko shape function

$E_{i}$ : Young's modulus of $i$ th step

$G_{i}$ : shear module of $i$ th step

$I_{i}$ : moment of inertia of $i$ th step

$J R i$ : inertia ratio with respect to first mass

$J_{i}$ : rotary inertia of concentrated mass

$m_{i}$ : mass distribution for $i$ th stage

$M_{i}$ : magnitude of $i$ th concentrated mass

$M R i$ : mass ratio with respect to first mass

$P$ : follower force

$R_{i}$ : rotary inertia parameter

$t$ : time

$w(x, t)$ : beam displacement

$x$ : $x$ coordinate

$y: y$ coordinate

$\mu_{i}$ : dimensionless concentrated mass

$\phi(x, t)$ : rotation in the $x-z$ plane

$\rho_{i}$ : density of $i$ th step

$L$ : beam length

Subscripts

$i$ : the $i$ th step

$t$ : first derivative with respect to time

\section{Introduction}

The instability of aerospace structures under the tangential and lateral thrust forces generated by their rocket or jet engines is of vital importance and is the interest of many

(C) 2012 The Japan Society for Aeronautical and Space Sciences researchers. In general, forces acting on a structure can be divided into conservative and non-conservative types. The follower force is a typical example of non-conservative forces. When a structure is under a constant follower force whose direction changes along the deformation of the structure, it may experience static or dynamic instability. The static instability, divergence, happens when transverse natural frequencies merge into zero. The dynamic instability, flutter, is associated with the coalescence of two natural frequencies into each other. Many researchers have studied the instability of a free-free beam subjected to a follower force as a mathematical simulation of flying flexible rockets, missiles or launch vehicles. Beal ${ }^{1)}$ investigated the stability of a uniform free-free beam under a controlled constant and pulsating follower force. For the case of constant thrust without a directional control system, he showed that the critical thrust magnitude is associated with the coalescence of the two lowest bending frequencies. In addition, with the control system included, he found that the critical thrust magnitude corresponds to the lowest frequency when it tends to zero. Finally, for the case of pulsating thrusts, he concluded that longitudinal stiffness plays an important role by showing instabilities caused by variations in the fundamental longitudinal beam frequency. Matsumoto and Mote ${ }^{2)}$ discussed the effect of a time delay in thrust vector control. Mote ${ }^{3)}$ and Barsoum $^{4)}$ used the extended Hamilton principle and finite element method to solve the problem. Peters and $\mathrm{Wu}^{5)}$ studied the lateral stability of a free-free column subjected to axial thrust with a control system. They concluded that, under the condition of non-direction control of the follower force, a pair of zero eigenvalues exists for all forces with an eigenfunction of first-order corresponding to rigid-body translation and an eigenfunction of second-order rank corresponding to the rigid-body rotation. $\mathrm{Wu}^{6}$ investigated the 


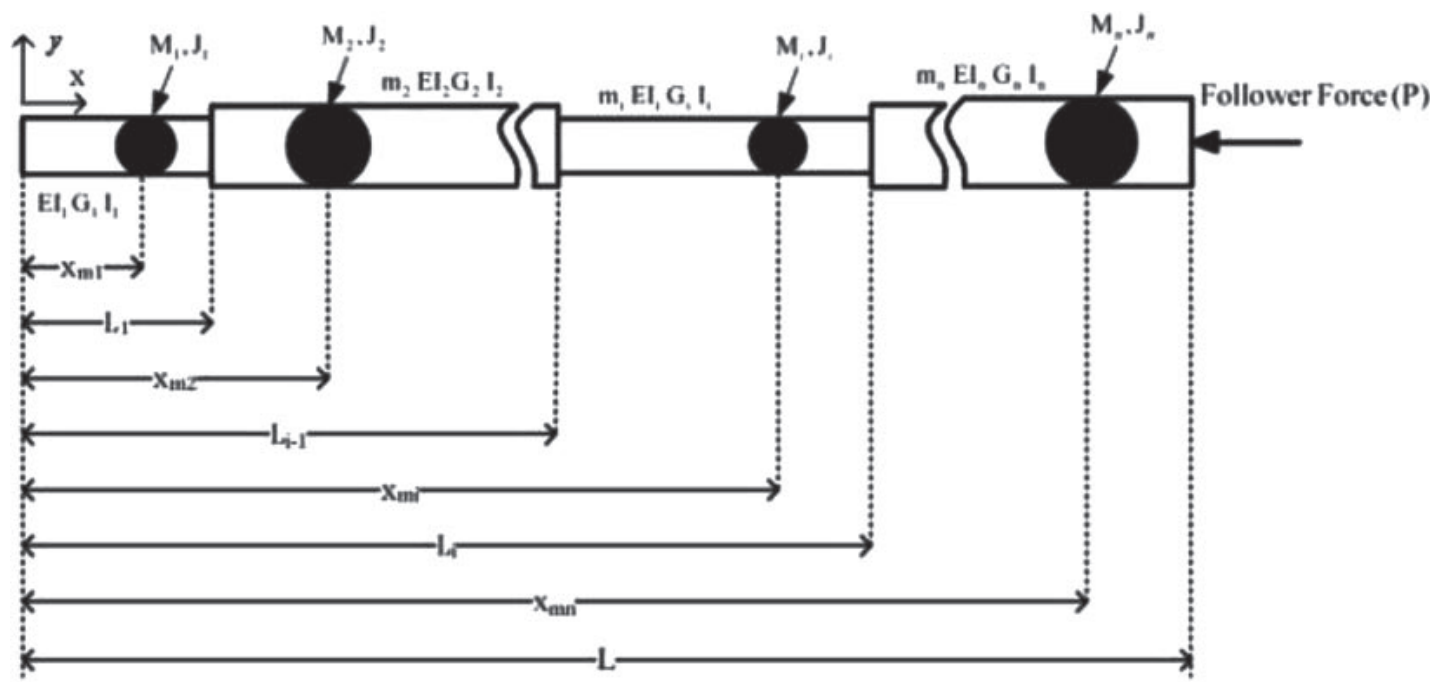

Fig. 1. A non-uniform, free-free beam composed of $n$-uniform beam segments and carrying $n$-lumped masses $M_{i}$ (with rotary inertia $J_{i}$ ) with optional positions by varying segment length.

relation between the critical load and eigencurves using the finite element method. He concluded that the magnitude and location of a concentrated mass improves the stability characteristics of a missile subjected to follower force.

Park and Mote $^{7)}$ studied a free-free Euler-Bernoulli beam, transporting a concentrated mass with rotary and transverse inertia under an end thrust. They investigated that both divergence and flutter instabilities can occur over the range of beam models examined. They showed that the location and magnitude of the additional mass, and the location and gain of the follower force direction sensor allow the follower force magnitude to be maximized, enabling stable transverse motion of the beam. Kirillov and Seyranian ${ }^{8)}$ studied mass distribution and stiffness optimization of a beam moving in space under a tangential end force. Park ${ }^{9)}$ studied the dynamic stability of a free-free Timoshenko beam under a controlled constant follower force, at which time the effects of rotary inertia and shear deformation on the stability of the beam were surveyed.

Kim and $\mathrm{Choo}^{10)}$ analyzed the dynamic stability of a freefree Timoshenko beam with a concentrated mass subjected to a pulsating follower force. The effects of axial location and translation inertia of the concentrated mass were studied. Yoon and $\mathrm{Kim}^{11)}$ analyzed the dynamic stability of a spinning beam subjected to a pulsating thrust. Leipholz and Piche ${ }^{12)}$ studied the effect of weight and follower forces on the stability of elastic rods using a two-term Galerkin approximation. Pradhan and Datta ${ }^{13)}$ studied the effect of rocket mass and an intermediate mass on the critical flutter load of a free-free beam. Recently, Irani and Kavianipour ${ }^{14}$ ) studied bipartite beams with a flexible joint under follower and transversal forces. They analyzed the effects of the characteristics of a flexible joint on beam instability using the maximum bearable propulsion force. They investigated if increasing the follower force or transversal force will, in turn, raise the IMU vibration of the model. Consequently, it causes a destructive phenomenon in the control system of the aerospace structure.
This paper presents a solution that can simulate many aerospace structures, such as lunch vehicles, with various stages varying in typical properties using the Timoshenko beam theory. The changes in mass distribution and beam stiffness result in variations in the dynamic and static instability regime of the free-free beam subjected to follower force.

\section{Formulation}

A sketch of the non-uniform, free-free beam studied in this paper is shown in Fig. 1. The model includes a number of uniform beam segments having different mass distribution, section and material properties. Additionally, the axial coordinate $x$ is shown in Fig. 1. In this research, the effects of aerodynamic forces and structural damping are ignored.

\subsection{Energy equation and external work}

For this beam, the strain energy $U$ is given by:

$U=\sum_{i=1}^{n} \frac{1}{2} \int_{L_{i-1}}^{L_{i}} E I_{i} \phi_{x}{ }^{2} \mathrm{~d} x+\frac{1}{2} \int_{L_{i-1}}^{L_{i}} k A_{i} G_{i}\left(w_{x}-\phi\right)^{2} \mathrm{~d} x$

where $n$ is the number of segments, $E I_{i}$ is bending stiffness, $k A_{i} G_{i}$ is shear stiffness, $\phi$ is rotation in the $x-z$ plane, $w_{x}$ is the first derivative of displacement in respect to $x, \phi_{x}$ is the first derivative of $\phi$ in respect to $x$, and $L_{i}$ is the coordinate of the $i$ th segment end point. This equation is formed by series because the total strain energy is the sum of the calculated values for each segment.

The total kinetic energy $T$ of the beam model can be calculated by summing up the kinetic energies of all segments with their concentrated mass. Therefore, the total kinetic energy is written as follows:

$$
\begin{aligned}
T= & \sum_{i=1}^{n}\left(\frac{1}{2} \int_{L_{i-1}}^{L_{i}} m_{i} w_{t}^{2} \mathrm{~d} x+\frac{1}{2} \int_{L_{i-1}}^{L_{i}} \rho_{i} I_{i} \phi_{t}{ }^{2} \mathrm{~d} x\right) \\
& +\sum_{i=1}^{n}\left(\left.\frac{1}{2} M_{i} w_{t}{ }^{2}\right|_{x=x_{M_{i}}}+\left.\frac{1}{2} J_{i} \phi_{t}{ }^{2}\right|_{x=x_{M_{i}}}\right),
\end{aligned}
$$


where $m_{i}, \rho_{i}$ and $I_{i}$ are the distributed mass, density and moment of inertia of the $i$ th segment, respectively and $M_{i}$ and $J_{i}$ are the magnitude and rotary inertia of concentrated mass, respectively, in the $i$ th segment.

The conservative part of the follower force is denoted by axial force. The axial force accelerates the system as follows:

$$
a=\frac{P-M^{\prime} g}{M^{\prime}}
$$

where $a$ is the acceleration of the beam, $P$ is the magnitude of the follower force and $M^{\prime}$ is the total mass. $M^{\prime}$ can be expressed as:

$$
M^{\prime}=\sum_{i=1}^{n}\left(M_{i}+m_{i} l_{i}\right)
$$

Therefore, the distributed axial force $\hat{P}(x)$ is:

$$
\begin{aligned}
\hat{P}_{i}(x)= & \left(\frac{P}{M^{\prime}}\right)\left[m_{i} x-m_{i} L_{i-1}+M_{i} H\left(x-x_{M_{i}}\right)\right. \\
& \left.+\sum_{j=1}^{i}\left(m_{j-1} l_{j-1}+M_{j-1}\right)\right],
\end{aligned}
$$

where $H$ is the Heaviside function and $l_{i}$ is the length of the $i$ th segment. Additionally, the external conservative work $W_{c}$ done by the axial force is described as follows:

$$
\begin{aligned}
W_{c}= & \sum_{i=1}^{n} \frac{1}{2} \int_{L_{i-1}}^{L_{i}}\left(\frac{P}{M^{\prime}}\right)\left[m_{i} x-m_{i} L_{i-1}+M_{i} H\left(x-x_{M_{i}}\right)\right. \\
& \left.+\sum_{j=1}^{i}\left(m_{j-1} l_{j-1}+M_{j-1}\right)\right] w_{x}^{2} \mathrm{~d} x
\end{aligned}
$$

However, the non-conservative work done by the follower force with respect to the directional control system is described by:

$$
\delta W_{N C}=-\left.\left.P \phi\right|_{x=L} \delta w\right|_{x=L}
$$

The extended Hamilton's principle for the non-conservative system can also be expressed by:

$$
\int_{t 1}^{t 2}\left(\delta\left(T-U+W_{C}\right)\right) \mathrm{d} t+\int_{t 1}^{t 2} \delta W_{N C} \mathrm{~d} t=0
$$

To simplify the governing equation, the following dimensionless parameters are introduced:

$$
\begin{aligned}
\xi & =\frac{x}{L}, \quad \xi_{(. .)}=\frac{x_{(. .)}}{L}, \quad \eta=\frac{w}{L}, \quad \lambda_{(. .)}=\frac{L_{(. .)}}{L}, \quad \hat{\lambda}_{i}=\frac{l_{i}}{L}, \\
e_{i} & =\frac{E_{i} I_{i}}{E_{1} I_{1}}, \quad v_{i}=\frac{m_{i}}{m_{1}} \\
\tau & =t \frac{1}{L^{2}} \sqrt{\frac{E_{1} I_{1}}{m_{1}}}, \quad S_{i}=\left(\frac{k G_{i} A_{i} L^{2}}{E_{1} I_{1}}\right), \quad r_{i}=\frac{\rho_{i}}{\rho_{1}}, \quad R_{i}=\frac{I_{i}}{A_{1} L^{2}} \\
\beta_{i} & =\frac{j_{i}}{m_{1} L^{3}}, \quad \mu_{i}=\frac{M_{i}}{m_{1} L}, \quad Q=\frac{P L^{2}}{E_{1} I_{1}}
\end{aligned}
$$

where $S_{i}$ and $R_{i}$ are shear deformation and rotary inertial parameter, respectively. By substituting Eqs. (1), (2), (6), (7) and (9) into Eq. (8), the governing equation can be expressed as:

$$
\begin{aligned}
& \int_{\tau_{1}}^{\tau_{2}}\left\{\sum_{i=1}^{n}\left(v_{i} \int_{\lambda_{i-1}}^{\lambda_{i}} \eta_{\tau} \delta \eta_{\tau} \mathrm{d} \xi+r_{i} R_{i} \int_{\lambda_{i-1}}^{\lambda_{i}} \phi_{\tau} \mathrm{d} \xi\right)+\sum_{i=1}^{n}\left(\left.\mu_{i} \eta_{\tau} \delta \eta_{\tau}\right|_{\xi=\xi_{M_{i}}}+\left.\beta_{i} \phi_{\tau} \delta \phi_{\tau}\right|_{\xi=\xi_{M_{i}}}\right)\right. \\
& \quad-\sum_{i=1}^{n}\left(\int_{\lambda_{i-1}}^{\lambda_{i}} e_{i} \phi_{\xi} \delta \phi_{\xi} \mathrm{d} \xi+\int_{\lambda_{i-1}}^{\lambda_{i}} S_{i}\left(\eta_{\xi}-\phi\right) \delta\left(\eta_{\xi}-\phi\right) \mathrm{d} \xi\right) \\
& \left.\quad+\sum_{i=1}^{n} \int_{\lambda_{i-1}}^{\lambda_{i}} \frac{Q}{\mu_{\text {total }}}\left(v_{i}\left(\xi-\lambda_{i-1}\right)+\mu_{i} H\left(\xi-\xi_{M_{i}}\right)+\mu_{\text {start }_{i}}\right) \eta_{\xi} \delta \eta_{\xi} \mathrm{d} \xi-\left.Q\left(\left.\phi\right|_{\xi=\lambda}\right) \delta \eta\right|_{\xi=\lambda}\right\} \mathrm{d} \tau=0
\end{aligned}
$$

In order to use the finite element method, each stage subdivides into $N_{i}$ elements. In each segment, the length of the elements is equal, but may vary from those of an adjacent segment.

For convenience, a local coordinate is defined as follows:

$$
\xi=\lambda_{i-1}+\Delta_{i}(m-1)+\Delta_{i} \tilde{\xi}_{i}
$$

where $\Delta_{i}=\left(\lambda_{i}-\lambda_{i-1}\right) / N_{i}$ and $m$ is the number of elements in the $i$ th segment.

Therefore, Eq. (10) can be changed to:

$$
\int_{\tau_{1}}^{\tau_{2}}\left\{\frac{Q}{\mu_{\text {total }}} \sum_{i=1}^{n} \sum_{m=1}^{N_{i}}\left(\frac{1}{\Delta_{i}} \int_{0}^{1}\left(v_{i}\left(\Delta_{i}(m-1)+\Delta_{i} \tilde{\xi}_{i}\right)+\mu_{i} H\left(\xi-\xi_{M_{i}}\right)+\mu_{\text {start }_{i}}\right) \mathrm{d} \tilde{\xi} \eta_{\tilde{\xi}} \delta \eta_{\tilde{\xi}}\right)\right.
$$




$$
\begin{aligned}
& +\sum_{i=1}^{n} \sum_{m=1}^{N_{i}} \Delta_{i} \int_{0}^{1}\left(v_{i} \eta_{\tau} \delta \eta_{\tau}+r_{i} R_{i} \phi_{\tau} \delta \phi_{\tau}\right) \mathrm{d} \tilde{\xi}+\sum_{i=1}^{n}\left(\left.\mu_{i} \eta_{\tau} \delta \eta_{\tau}\right|_{\xi=\xi_{M_{i}}}+\left.\beta_{i} \phi_{\tau} \delta \phi_{\tau}\right|_{\xi=\xi_{M_{i}}}\right) \\
& \left.-\sum_{i=1}^{n} \sum_{m=1}^{N_{i}} \Delta_{i} \int_{0}^{1}\left(\frac{1}{\Delta_{i}^{2}} e_{i} \phi_{\tilde{\xi}} \delta \phi_{\tilde{\xi}}+S_{i}\left(\frac{1}{\Delta_{i}} \eta_{\tilde{\xi}}-\phi\right) \delta\left(\frac{1}{\Delta_{i}} \eta_{\tilde{\xi}}-\phi\right)\right) \mathrm{d} \tilde{\xi}-\left.Q\left(\left.\phi\right|_{\xi=\lambda}\right) \delta \eta\right|_{\xi=\lambda}\right\} \mathrm{d} \tau=0
\end{aligned}
$$

Assume the solution of Eq. (12) has the following form:

$$
\begin{aligned}
& \eta^{i}(\xi, \tau)=A^{i}(\xi) V(\tau) \\
& \phi^{i}(\xi, \tau)=B^{i}(\xi) V(\tau),
\end{aligned}
$$

where $A^{i}(\xi)$ and $B^{i}(\xi)$ are the Timoshenko shape functions. ${ }^{15)}$ To clarify Eq. (12), it is divided into four terms, $a, b, c$ and $d$, which are defined as below:

$$
\begin{aligned}
a= & \sum_{i=1}^{n} \sum_{m=1}^{N_{i}}\left(\Delta_{i} \int_{0}^{1}\left(v_{i} \eta_{\tau \tau} \delta \eta+r_{i} R_{i} \phi_{\tau \tau} \delta \phi\right) \mathrm{d} \tilde{\xi}\right) \\
& -\sum_{i=1}^{n} \sum_{m=1}^{N_{i}} \Delta_{i} \int_{0}^{1}\left(\frac{1}{\Delta_{i}^{2}} e_{i} \phi_{\xi} \delta \phi_{\tilde{\xi}}\right. \\
& \left.+S_{i}\left(\frac{1}{\Delta_{i}} \eta_{\tilde{\xi}}-\phi\right) \delta\left(\frac{1}{\Delta_{i}} \eta_{\tilde{\xi}}-\phi\right)\right) \mathrm{d} \tilde{\xi} \\
b= & \frac{Q}{\mu_{\text {total }}} \sum_{i=1}^{n} \sum_{m=1}^{N_{i}}\left(\frac { 1 } { \Delta _ { i } } \int _ { 0 } ^ { 1 } \left(v_{i}\left(\Delta_{i}(m-1)+\Delta_{i} \tilde{\xi}_{i}\right)\right.\right. \\
& \left.\left.+\mu_{i} H\left(\xi-\xi_{M_{i}}\right)+\mu_{\text {start }_{i}}\right) \eta_{\tilde{\xi}} \delta \eta_{\tilde{\xi}} \mathrm{d} \tilde{\xi}\right) \\
c= & \sum_{i=1}^{n}\left(\left.\mu_{i} \eta_{\tau \tau} \delta \eta\right|_{\xi=\xi_{M_{i}}}+\left.\beta_{i} \phi_{\tau \tau} \delta \phi\right|_{\xi=\xi_{M_{i}}}\right) \\
d= & -\left.Q\left(\left.K_{s} \phi\right|_{\xi=\xi_{s}}+\left.\phi\right|_{\xi=\lambda}\right) \delta \eta\right|_{\xi=\lambda}
\end{aligned}
$$

Hence,

$$
a+b+c+d=0 .
$$

Applying all of the above, the governing equation can be reduced to:

$$
[M] \ddot{V}+[K] V=0,
$$

where $[K]$ and $[M]$ are stiffness and mass matrix, respectively. For part $(a)$ :

$$
\begin{aligned}
a= & \sum_{i=1}^{n} \sum_{m=1}^{N_{i}}\left(\Delta _ { i } \int _ { 0 } ^ { 1 } \left(v A^{\mathrm{T}}(\xi) A(\xi) V_{\tau \tau} \delta V\right.\right. \\
& \left.\left.+r_{i} R_{i} B^{\mathrm{T}}(\xi) B(\xi) V_{\tau \tau} \delta V\right) \mathrm{~d} \tilde{\xi}\right) \\
& -\sum_{i=1}^{n} \sum_{m=1}^{N_{i}} \Delta_{i} \int_{0}^{1}\left(\frac{1}{\Delta_{i}^{2}} e_{i} B_{\tilde{\xi}}^{\mathrm{T}}(\xi) B_{\tilde{\xi}}(\xi) V \delta V\right. \\
& \left.+S_{i}\left(\frac{1}{\Delta_{i}} A_{\tilde{\xi}}^{\mathrm{T}}(\xi)-B^{\mathrm{T}}(\xi)\right)\left(\frac{1}{\Delta_{i}} A_{\tilde{\xi}}(\xi)-B(\xi)\right) V \delta V\right) \mathrm{d} \tilde{\xi}
\end{aligned}
$$

Therefore, stiffness and mass matrix develop as follows: $a=\sum_{i=1}^{n} \sum_{j=1}^{N_{i}} \delta V^{i}\left(M_{A_{1}} V_{\tau \tau}+M_{A_{2}} V_{\tau \tau}+K_{A_{1}} V+K_{A_{2}} V\right)$, where

$$
\begin{aligned}
M_{a_{1}} & =\Delta_{i} v_{i} \int_{0}^{1} A^{\mathrm{T}}(\tilde{\xi}) A(\tilde{\xi}) \mathrm{d} \tilde{\xi} \\
M_{a_{2}} & =\Delta_{i} r_{i} R_{i} \int_{0}^{1} B^{\mathrm{T}}(\tilde{\xi}) B(\tilde{\xi}) \mathrm{d} \tilde{\xi} \\
K_{a_{1}} & =\frac{1}{\Delta_{j}} e_{i} \int_{0}^{1} B_{\tilde{\xi}}^{\mathrm{T}}(\tilde{\xi}) B_{\tilde{\xi}}(\tilde{\xi}) \mathrm{d} \tilde{\xi}_{i} \\
K_{a_{2}} & =S_{j} \frac{1}{\Delta_{j}} \int_{0}^{1} C^{\mathrm{T}}(\tilde{\xi}) C(\tilde{\xi}) \mathrm{d} \tilde{\xi} \\
C(\tilde{\xi}) & =\frac{1}{\Delta_{i}} A_{\tilde{\xi}}(\tilde{\xi})-B(\tilde{\xi})
\end{aligned}
$$

There is a similar procedure for part $(b)$ of the equation:

$$
\begin{aligned}
b= & \frac{Q}{\mu_{\text {total }}} \sum_{i=1}^{n} \sum_{m=1}^{N_{i}}\left(\frac { 1 } { \Delta _ { i } } \int _ { 0 } ^ { 1 } \left(v_{i}\left(\Delta_{i}(m-1)+\Delta_{i} \tilde{\xi}_{i}\right)\right.\right. \\
& \left.\left.+\mu_{i} H\left(\xi-\xi_{M_{i}}\right)+\mu_{\text {start }_{i}}\right) A_{\tilde{\xi}}^{\mathrm{T}}(\xi) A_{\tilde{\xi}}(\xi) V \delta V \mathrm{~d} \tilde{\xi}\right) \\
b= & \sum_{i=1}^{n} \sum_{m=1}^{N_{i}} \delta V(\tau)\left[K_{b_{1}} V(\tau)+K_{b_{2}} V(\tau)\right],
\end{aligned}
$$

where

$$
\begin{aligned}
K_{b_{2}}= & \frac{Q}{\mu_{\text {total }}}\left(\int_{0}^{1} v_{i} \tilde{\xi}_{i} A_{\tilde{\xi}}^{\mathrm{T}}(\xi) A_{\tilde{\xi}}(\xi) \mathrm{d} \tilde{\xi}\right) \\
K_{b_{1}}= & \frac{Q}{\mu_{\text {total }}} \frac{1}{\Delta_{i}} \int_{0}^{1}\left(v_{i}\left(\Delta_{i}(m-1)\right)+\mu_{i} H\left(\xi-\xi_{M_{i}}\right)+\mu_{\text {start }_{i}}\right) \\
& \times A_{\tilde{\xi}}^{\mathrm{T}}(\xi) A_{\tilde{\xi}}(\xi) \mathrm{d} \tilde{\xi} .
\end{aligned}
$$

Part $(c)$ of the equation contains the effect of concentrated masses.

$$
\begin{aligned}
c= & \sum_{i=1}^{n}\left(\left.\mu_{i} A^{\mathrm{T}}(\xi) A(\xi)\right|_{\xi=\xi_{M_{i}}} V_{\tau \tau} \delta V\right. \\
& \left.+\left.\beta_{i} B^{\mathrm{T}}(\xi) B(\xi)\right|_{\xi=\xi_{M_{i}}} V_{\tau \tau} \delta V\right)
\end{aligned}
$$

where

$$
\begin{aligned}
& M_{c_{1}}=\left.\mu_{i} A^{\mathrm{T}}(\xi) A(\xi)\right|_{\xi=\xi_{M_{i}}} \\
& M_{c_{2}}=\left.\beta_{i} B^{\mathrm{T}}(\xi) B(\xi)\right|_{\xi=\xi_{M_{i}}} .
\end{aligned}
$$

Finally, part $(d)$ can be written as follows:

$$
d=\left(\left[K_{d_{1}}\right]\right) V(\tau) \delta V
$$

where

$$
K_{d_{1}}=-Q B(1)_{\xi=\lambda} A^{\mathrm{T}}(1)_{\xi=\lambda} .
$$

To calculate the total mass matrix, one should add those of parts $(a)$ and $(c)$ together.

$$
[M]=\left[M_{a_{1}}\right]+\left[M_{a_{2}}\right]+\left[M_{c_{1}}\right]+\left[M_{c_{2}}\right]
$$




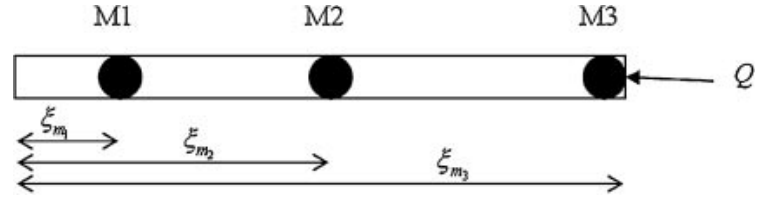

Fig. 2. Uniform Timoshenko beam with three concentrated masses subjected to follower force.

Acting similarly for the stiffness matrix:

$$
\begin{aligned}
{[K]=} & {\left[K_{a_{1}}\right]+\left[K_{a_{2}}\right]+\left[K_{b_{1}}\right]+\left[K_{b_{2}}\right] } \\
& +\left[K_{c_{1}}\right]+\left[K_{c_{2}}\right]+\left[K_{d_{1}}\right]+\left[K_{d_{2}}\right]
\end{aligned}
$$

\section{Results and Discussion}

\subsection{Stability analysis of the beam}

Next, assume that $V(\tau)=e^{i \omega \tau}$, as result, the deflection $(\eta)$ and rotation of the beam $(\phi)$ are described as follows:

$$
\begin{gathered}
\eta(\xi, \tau)=A(\xi) e^{i \omega \tau} \\
\phi(\xi, \tau)=B(\xi) e^{i \omega \tau},
\end{gathered}
$$

where $\omega$ is the non-dimensional natural frequency of the beam. Accordingly, Eq. (19) can be changed to:

$$
\left([K]-\omega^{2}[M]\right) V=0
$$

Beal $^{1)}$ described how dynamic and static instability are defined by the amount and sign of $\omega$ with respect to follower force.

\subsection{Effect of three concentrated masses on dynamic and static instability characteristics of uniform} a beam

A uniform beam with three concentrated masses is a mathematical model of some aerospace structures. In this case, three equal segments are assumed, each having a concentrated mass.

This model is illustrated in Fig. 2.

In this figure, the position of each concentrated mass is defined by $\xi_{m_{i}}$.

Each concentrated mass contains transverse inertia $\left(M_{i}\right)$ and rotary inertia $\left(J_{i}\right)$, which are defined in dimensionless form as $\left(\mu_{i}\right)$ and $\left(\beta_{i}\right)$, respectively.

The critical follower forces were calculated for a uniform beam having 30 elements in the modeling based on a convergence study. The critical follower force for this configuration was plotted versus change in position of the first mass, as shown in Fig. 3.

These results are plotted using different conditions for rotary inertia of the beam and concentrated masses.

In the governing equation, by setting the rotary inertia of the beam and concentrated masses equal to zero $\left(R=\beta_{1}=\right.$ $\beta_{2}=\beta_{3}=0$ ), the critical follower force rises to 85.4 at $\xi_{m_{1}}=0.24$. This part has static instability (divergence). After this point, the critical follower force decreases due to the change in type of stability to dynamic. When the first mass position passes $\xi_{m_{1}}=0.46$, the critical follower force increases again. Figure 4 illustrates that when the mass position is 0.18 , type of instability is static; in addition, by

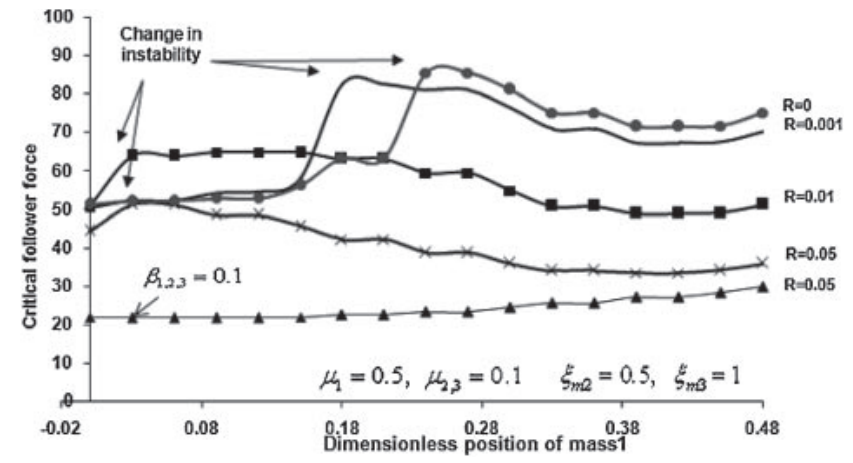

Fig. 3. Critical follower force versus dimensionless position of the first mass.

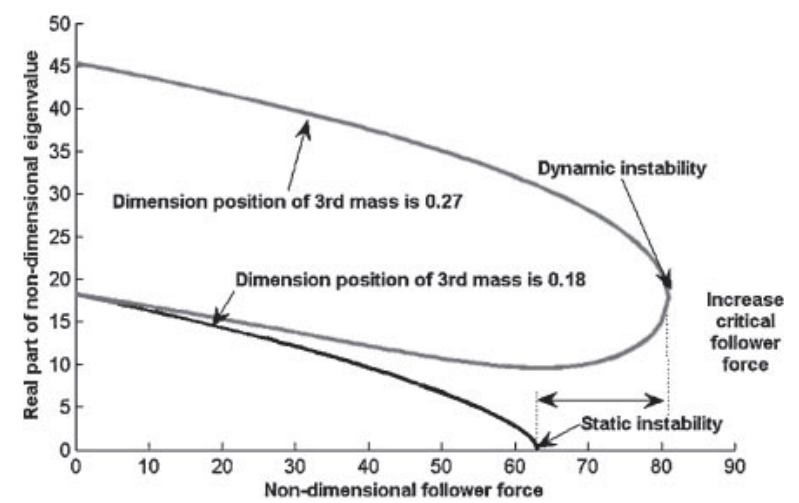

Fig. 4. Change in magnitude of critical follower force by changing the type of instability at $R=\beta_{1}=\beta_{2}=\beta_{3}=0$.

increasing mass position to 0.27 , type of instability changes to dynamic one and critical follower force rises significantly.

Furthermore, in reality, beam and concentrated masses have rotary inertia that significantly affects the critical follower force and type of instability.

In another case, the rotary inertia of the beam is set equal to 0.001 and rotary inertia of the concentrated masses is zero $\left(R=0.001, \beta_{1}=\beta_{2}=\beta_{3}=0\right)$. In comparison to the previous one, the critical follower force becomes larger when $\xi_{m_{1}}<0.24$. Furthermore it is concluded from Fig. 3 that, as the rotary inertia of the beam increases, the change in follower force decrease. In other words, when $R=\beta_{1}=\beta_{2}=$ $\beta_{3}=0$, the critical follower force changes between 85.4 and 50.1 , but when $R=0.05, \beta_{1}=\beta_{2}=\beta_{3}=0.01$, the critical follower force changes between 29.8 and 21.9 with respect to the position of the first mass. When $R=\beta_{1}=\beta_{2}=$ $\beta_{3}=0$, instability changes from static to dynamic but in the case of $R=0.05, \beta_{1}=\beta_{2}=\beta_{3}=0.01$, only dynamic instability remains.

In Fig. 3, the type of instability changes at $\xi_{m_{1}}=0.24$ when ignoring rotary inertia of the beam and concentrated masses. When $R=0.001, \beta_{1}=\beta_{2}=\beta_{3}=0$, the type of instability changes at $\xi_{m_{1}}=0.18$, but when $R=0.05$, $\beta_{1}=\beta_{2}=\beta_{3}=0$, static instability changes to dynamic instability at $\xi_{m_{1}}=0.03$. It can be concluded that, as the rotary inertia of the beam and concentrated masses increase, the type of instability changes as $\xi_{m_{1}}$ decrease. Additionally, by increasing the rotary inertia parameter of the beam, the 


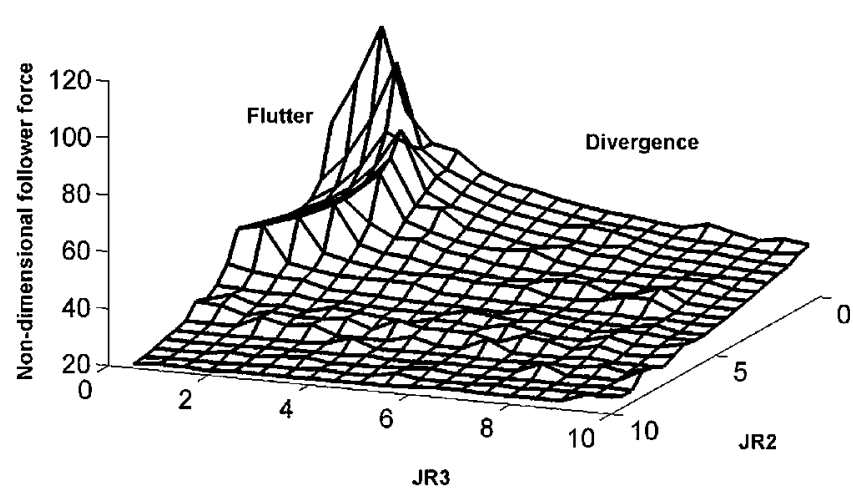

Fig. 5. Change in critical follower force versus concentrated mass rotary inertia ratio.

Table 1. Concentrated mass properties.

\begin{tabular}{lccl}
\hline First mass & $\mu_{1}=0.1$ & $\beta_{1}=0.01$ & $\xi_{m_{1}}=0.33$ \\
Second mass & $\mu_{2}=0.1$ & $J R 2$ & $\xi_{m_{2}}=0.66$ \\
Third mass & $\mu_{3}=0.1$ & $J R 3$ & $\xi_{m_{3}}=1$ \\
\hline
\end{tabular}

only dynamic instability is present. For example, when $R=0.05, \beta_{1}=\beta_{2}=\beta_{3}=0.01$, the system is subject to only dynamic instability.

Change in the magnitude of each mass with respect to the first one has an effect on system stability. The mass and shear deformation ratios of each mass (i.e., second and third masses) with respect to the first mass are defined as follows:

$$
\begin{array}{r}
M R i=\frac{\mu_{i}}{\mu_{1}} \\
J R i=\frac{\beta_{i}}{\beta_{1}}
\end{array}
$$

To analyze this model, the rotary inertia parameter of the beam has been ignored. $S$ is set to $10^{6}$, and it is assumed that the concentrated mass has the properties described in Table 1.

The critical follower force versus rotary inertia ratio of the concentrated masses is plotted in Fig. 5. In this figure, $J R 2$ changes between 0.1 and 10 . For example, when $J R 2=0.3$, the rotary inertia of the second concentrated mass must be $\beta_{2}=0.003$. In other words, $\beta_{2}$ and $\beta_{3}$ change between 0.001 and 0.1 . One can observe that, as the magnitude of $J R 3$ increases from 0.1 to 10 , the critical follower force reaches a specific point and then suddenly decreases. Changes in the type of stability play an important role. The domain of flutter and divergence is shown in Fig. 6. By comparing Fig. 5 and Fig. 6, it is evident that the maximum critical follower force occurs at lowest $J R 2$ value and $J R 3$ has reached the critical value to change the type of instability.

In the case of $J R 2=0.5$ and $J R 3=1.5$, the variation of dimensionless critical follower force versus mass ratio of the second concentrated mass (MR2) and mass ratio of the third concentrated mass is shown in Fig. 7.

The mass ratios of the second and third concentrated masses vary between 0.1 and 5 . As shown here, the magnitude of the critical follower force and the type of instability change with this variation.

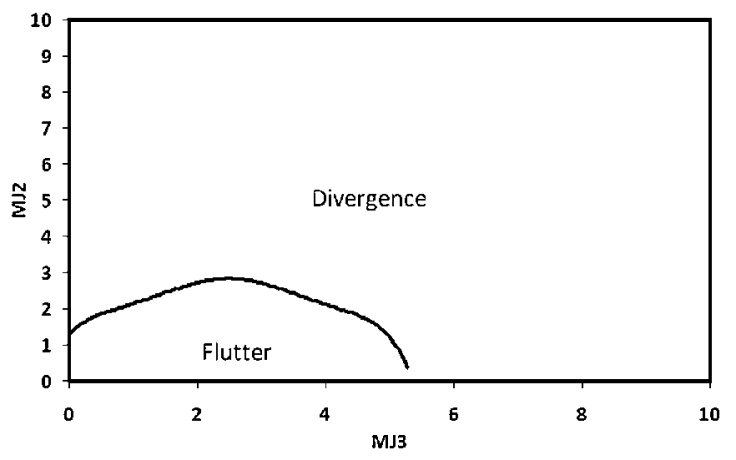

Fig. 6. Type of instability versus rotary inertia ratio.

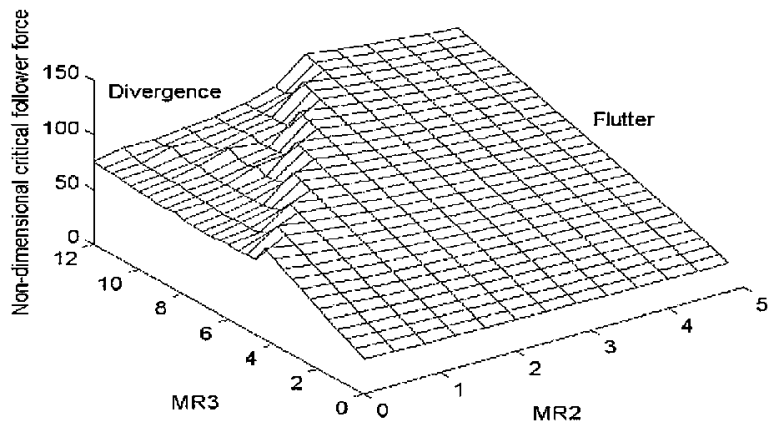

Fig. 7. Non-dimension follower force versus $M R 2$ and $M R 3$ at $J R 2=0.5$ and $J R 3=1.5$.

\subsection{Dynamic and static stability analysis of three- stepped beam without concentrated masses}

In this chapter, a beam is modeled as a cylindrical shell having a thickness much smaller than its diameter. Changes in diameter at each stage can cause the stiffness and mass properties to vary as given in these equations:

$$
\begin{aligned}
\frac{A_{i}}{A_{1}} & =\frac{D_{i}}{D_{1}}=A D i \\
\frac{I_{i}}{I_{1}} & =\left(\frac{D_{i}}{D_{1}}\right)^{3}=A D i^{3} \\
\frac{S_{i}}{S_{1}} & =\frac{A_{i}}{A_{1}}=\frac{D_{i}}{D_{1}}=A D i,
\end{aligned}
$$

where $D_{i}$ and $A D i$ are diameter and diameter ratio of the $i$ th stage with respect to the first stage, respectively.

The variation in critical dimensionless follower force versus diameter ratio of the second and third stages is shown in Fig. 8.

It is observed that when the values of $A D 3, A D 2$ increase, the critical follower force becomes larger. This is expected from a physical point of view because the diameter ratio and flexural rigidity $(E I)$ vary with respect to each other. Therefore, an increase in either of them can cause the beam stiffness and critical follower force to increase. This is true till $A D 3$ reaches 0.5 ; however from $A D 3=0.5$ to $A D 3=2$, the follower force decreases. After this point any increase in $A D 3$ will result in increase in an critical follower force again. These various changes in critical follower force are only due to the changes in type of instability. A stability diagram of the three-stepped beam is plotted in Fig. 9. 


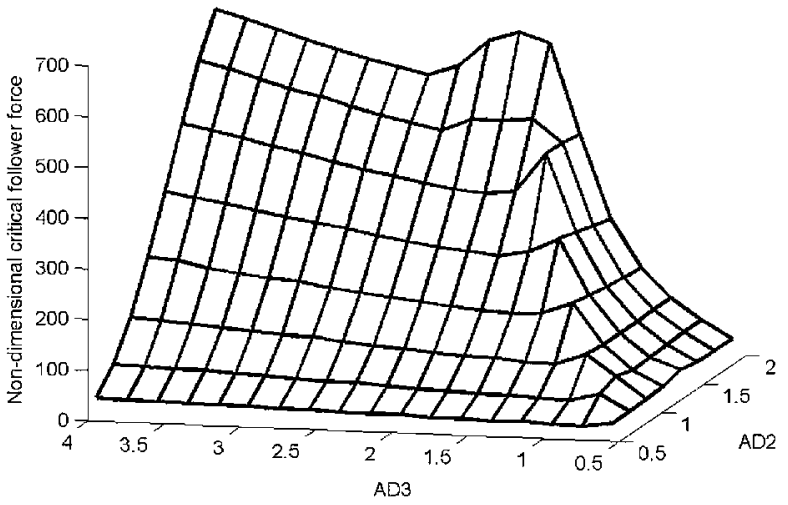

Fig. 8. Dimensionless follower force versus diameter ratio of second and third stage.

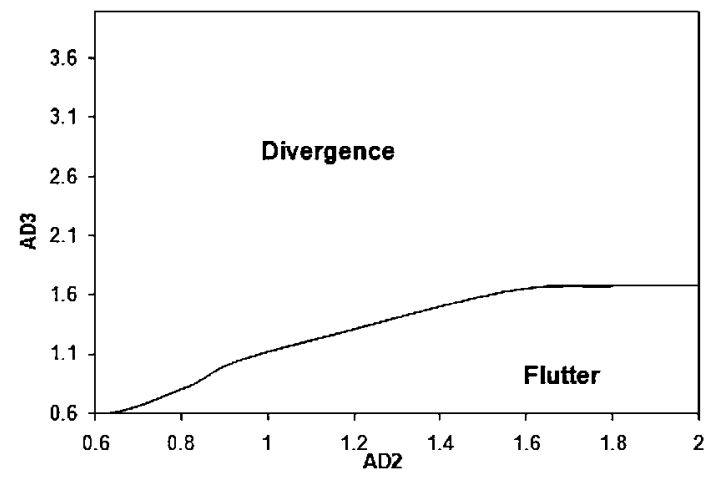

Fig. 9. Static and dynamic instability domain.

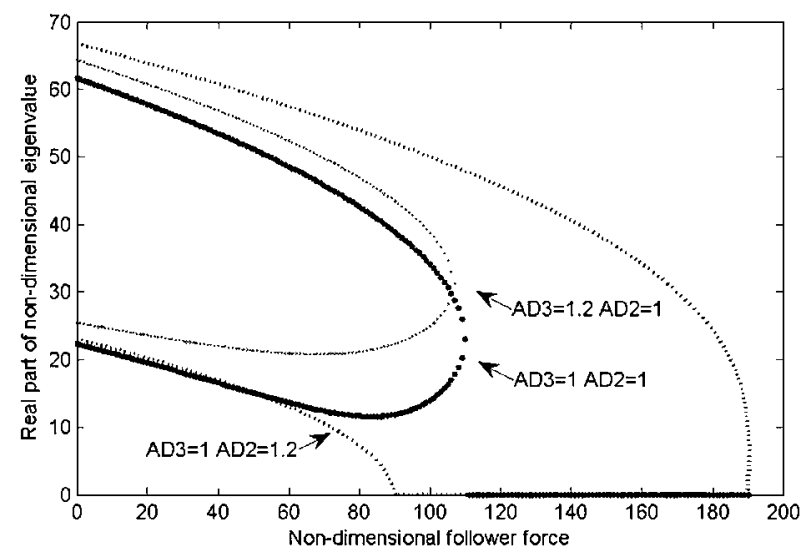

Fig. 10. Change in type of instability resulting from small change in diameter ratio.

It is depicted in Fig. 10 that a small change in the diameter ratio ( $A D 2$ and $A D 3$ ) causes the type of instability to change. It is also shown that the variety of beam section stiffness, mass distribution, shear deformation and rotary inertia affect the magnitude and type of instability significantly.

\subsection{Four-stepped beam without concentrated mass}

Similar to the previous section, a beam is modeled as a cylindrical shell which its thickness is much smaller than its diameter. Changes in diameter of each step can cause the stiffness and the mass properties vary by Eqs. (41)-(43).

The variation in critical dimensionless follower force versus $A D 3$ and $A D 4$ is shown in Fig. 11. The stability type

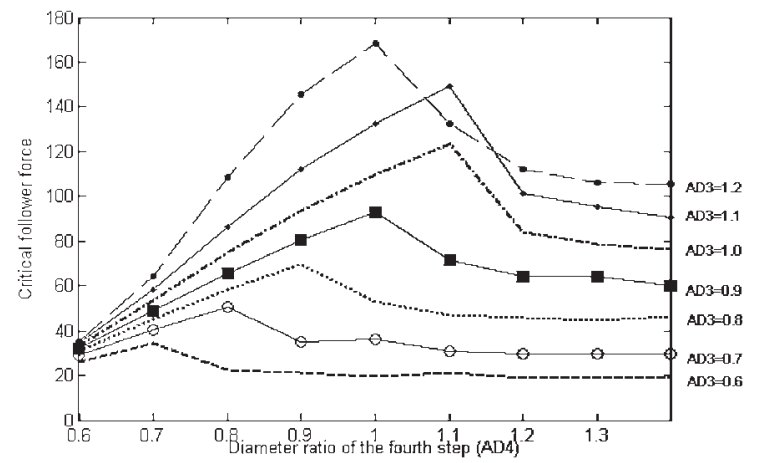

(a)

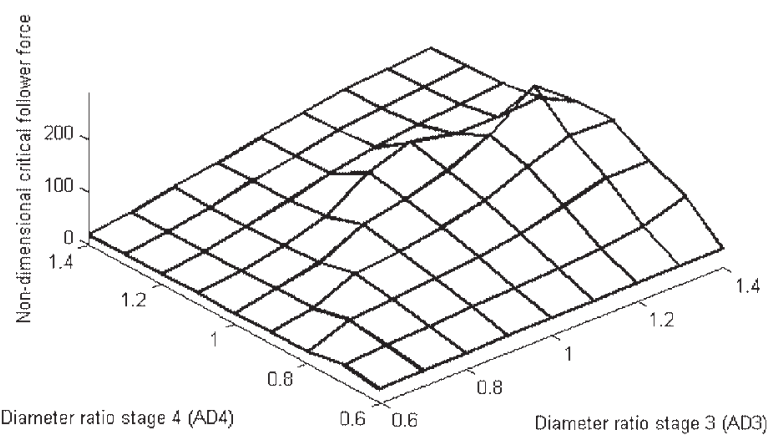

(b)

Fig. 11. Critical non-dimensional Follower Force versus $A D 3$ and $A D 4$ at $A D 1=1$ and $A D 2=1$.

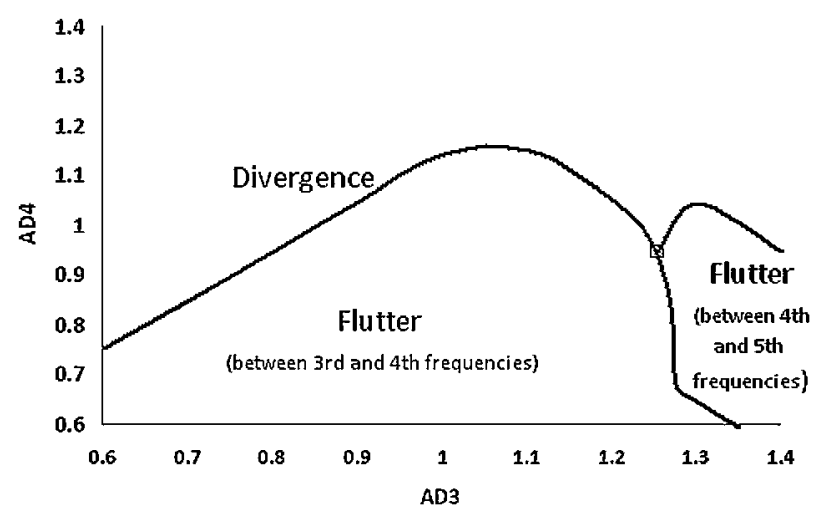

Fig. 12. Stability type versus $A D 3$ and $A D 4$ at $A D 1=1$ and $A D 2=1$.

versus $A D 3$ and $A D 4$ at $A D 1=A D 2=1$ is illustrated in Fig. 12.

It is observed that three types of instability could occur at the time in large amounts of the follower force. Divergence and flutter between the third and fourth frequencies are defined before hard (two rigid body motions are counted). The third one is the flutter associated with the coalescence of the fourth and the fifth frequencies. However, the third frequency has no effect on stability. These three types of instability are shown in Fig. 13.

Although $A D 1$ and $A D 2$ are constant, by applying a small change in the diameter ratio of the third and fourth steps, the critical follower force changes significantly. The change in the type of instability is the most important reason for changing the magnitude of the critical follower force. 


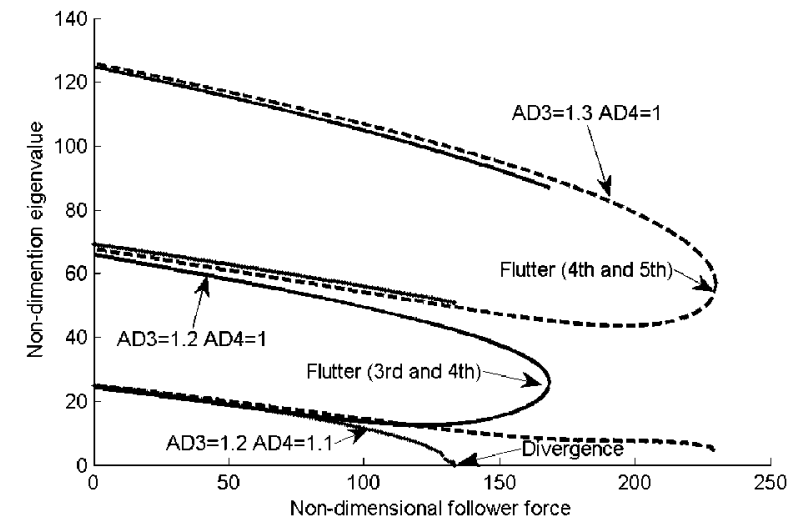

Fig. 13. Significant change in the follower force by applying a small change in diameter ratio $(A D 1=A D 2=1)$.

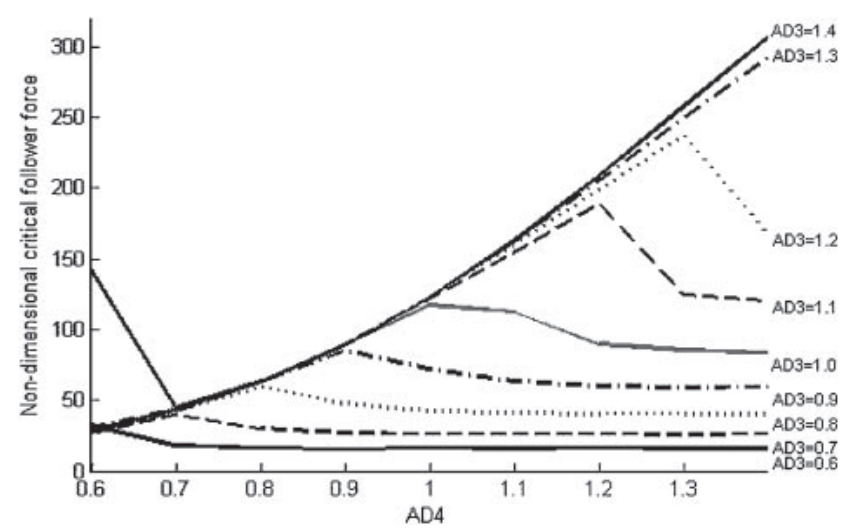

(a)

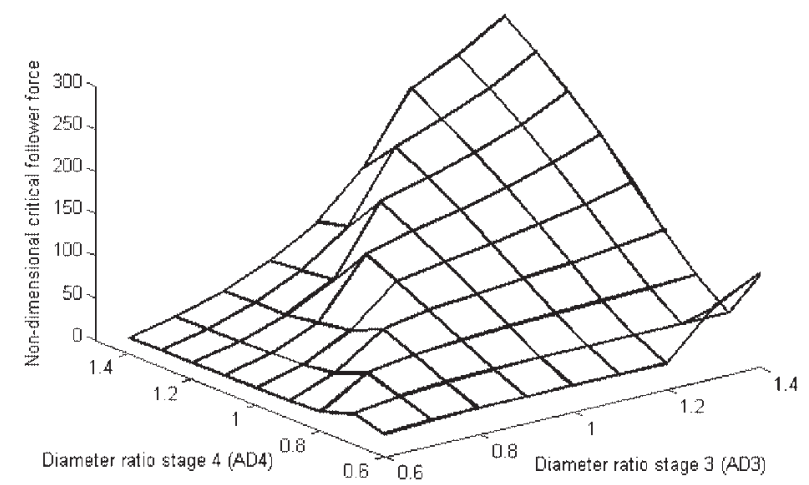

(b)

Fig. 14. Critical non-dimensional follower force versus $A D 3$ and $A D 4$ at $A D 1=1$ and $A D 2=1.4$.

For example, in Fig. 14 the critical follower force is 230.5 at $A D 3=1.3$ and $A D 4=1$. When $A D 3$ decreases only by an amount of 0.1 , the critical follower force becomes 168.4. Additionally when $A D 3=1.3$ and $A D 4=1.1$, the instability is divergence and the critical follower force changes to 133.3 .

In the case of $A D 2=1.4$, the critical follower force versus $A D 3$ and $A D 4$ is shown in Fig. 14. the diameter ratio of the third and fourth steps change from 0.6 to 1.4 , and the critical follower force varies between 19.3 and 294.8. The three types of instability described occur in this model, and this phenomenon is illustrated in Fig. 15. The domain

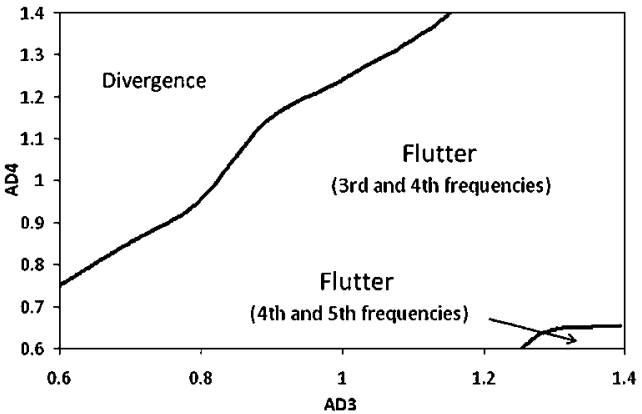

Fig. 15. Primary instability type versus $A D 3$ and $A D 4$ at $A D 2=1.4$.

Table 2. Concentrated mass properties.

\begin{tabular}{llll}
\hline First mass & $\mu_{1}=0.1$ & $\beta_{1}=0.01$ & $\xi_{m_{1}}=0.5$ \\
Second mass & $\mu_{2}=0.1$ & $\beta_{2}=0.01$ & $\xi_{m_{2}}=0.5$ \\
\hline
\end{tabular}

of the flutter between the fourth and fifth frequencies becomes smaller with respect to $A D 2=1$.

The type of instability and critical follower force are affected by the diameter ratios of these four steps. The variation in the diameter ratio of the beam makes it hard to predict its behavior when subjected to the follower force.

\subsection{Four-stepped beam with concentrated masses}

The effect of concentrated mass on the instability type and critical follower force has been studied in many references. In this paper, a four-stepped beam with two concentrated masses is modeled. The properties of these concentrated masses are shown in Table 2.

The critical dimensionless follower force versus $A D 3$ and $A D 4$ at $A D 2=1$ is plotted in Fig. 16. Additionally, the stability type versus $A D 3$ and $A D 4$ at $A D 1=A D 2=1$ is illustrated in Fig. 17. $A D 3$ and $A D 4$ change from 0.6 to 1.4, and it is observed that the critical follower force varies between 18.2 and 220.8. However, when $A D 3$ and $A D 4$ change without concentrated mass, the critical follower force varies between 19.3 and 230. At first it appears that the system is likely to be unstable, but when $A D 2=1, A D 3=1.4$ and $A D 4=1.2$, in the case of having no concentrated mass, the critical follower force is 126.8; whereas with two concentrated masses, the critical follower force becomes 220.8 .

It is concluded that the magnitude, location and number of concentrated masses may raise the value of the critical follower force.

On the other hand, three types of instability can occur when modeling the beam with no concentrated mass. Furthermore, two types of instability occur when carrying two concentrated masses.

\section{Conclusion}

The main purpose of this paper is to model a multi-stepped Timoshenko beam subjected to a follower force and study the effect of varying segment properties in terms of type of instability and critical follower force. For each case study, the results concluded are described below. 


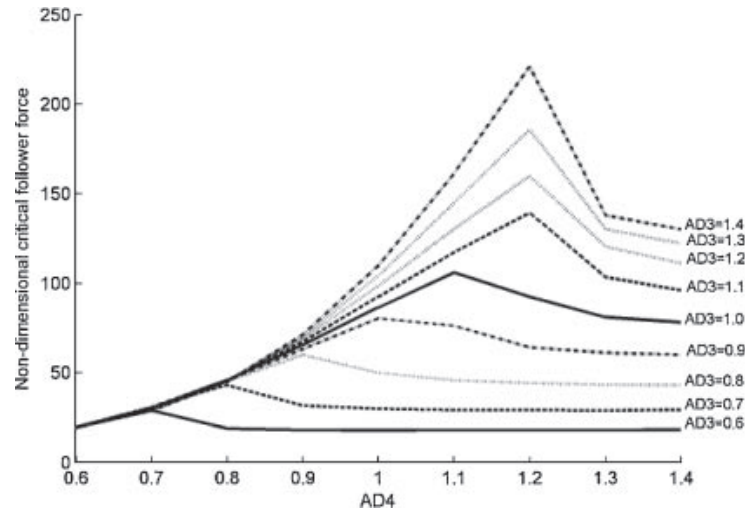

(a)

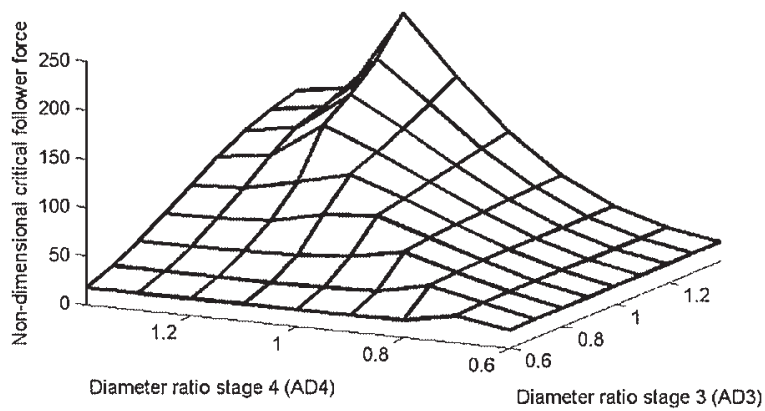

(b)

Fig. 16. Critical follower force versus $A D 3$ and $A D 4$ at $A D 2=1$ (with two concentrated masses).

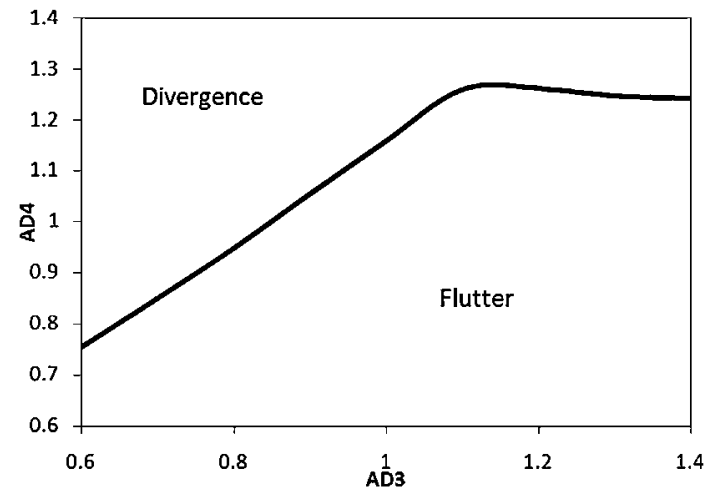

Fig. 17. Primary instability type versus $A D 3$ and $A D 4$ at $A D 2=1$ (with two concentrated masses).

Uniform beam with three concentrated masses: The variation in rotary inertia parameters of the beam and concentrated masses changes the type of instability, which in turn thoroughly alters the magnitude of the critical follower force.

Three-stepped beam: In the case of a beam without concentrated masses and direction control, the type of instability and magnitude of the follower force depend on the diam- eter ratio of the beam's stages, especially on the third stage. By increasing this dimensionless parameter, the type of instability changes from divergence to flutter. Critical follower force rises significantly.

Four-stepped beam: In this case, dynamic instability occurs not only when the third and forth frequencies are coalesced, but also when the forth and fifth frequencies act similarly.

Finally, it is concluded that, by applying a small change in diameter ratio for each stage, the type of instability and critical follower force may vary significantly. Therefore, the assumption of a uniform beam to study most aerospace structures such as launch vehicles could lead to an unrealistic analysis of instability. This study also introduces a new concept of parametric study to analyze the effects of the characteristics of multi-stage aerospace structures that could be considered during design.

\section{References}

1) Beal, T. R.: Dynamic Stability of a Flexible Missile under Constant and Pulsating Thrusts, AIAA J., 3, 3 (1965), pp. 486-494.

2) Matsumoto, G. Y. and Mote, C. D., Jr.: Time Delay Instabilities in Large Order Systems with Controlled Follower Force, J. Dyn. Syst. Meas. Contr., 94, 4 (1972), pp. 330-334.

3) Mote, C. D.: Nonconservative Stability by Finite Element, J. Eng. Mech. Div., Proceedings of the ASCE, 3 (1971), pp. 645-656.

4) Barsoum, R. S.: Finite Element Method Applied to Problem of Stability of a Nonconservative Systems, Int. J. Numer. Meth. Eng., 3 (1971), pp. 63-87.

5) Peters, D. A. and Wu, J. J.: Asymptotic Solutions to a Stability Problem, J. Sound Vib., 59, 4 (1978), pp. 591-610.

6) Wu, J. J.: Missile Stability Using Finite Elements-An Unconstrained Variational Approach, AIAA J., 14 (1976), pp. 313-319.

7) Park, Y. P. and Mote, C. D., Jr.: The Maximum Controlled Follower Force on a Free-Free Beam Carrying a Concentrated Mass, J. Sound Vib., 98, 2 (1985), pp. 247-256.

8) Kirillov, O. N. and Seyranian, A. P.: Optimization of Stability of a Flexible Missile under Follower Thrust, AIAA Paper 98-4969, 1998.

9) Park, Y. P.: Dynamic Stability of a Free Timoshenko Beam under a Controlled Follower Force, J. Sound Vib., 113, 3 (1987), pp. 407-415.

10) Kim, J.-H. and Choo, Y.-S.: Dynamic Stability of a Free-Free Timoshenko Beam Subjected to a Pulsating Follower Force, J. Sound Vib., 216, 4 (1998), pp. 623-636.

11) Yoon, S. J. and Kim, J. H.: A Concentrated Mass on the Spinning Unconstrained Beam Subjected to a Thrust, J. Sound Vib., 254, 4 (2002), pp. 621-634.

12) Leipholz, H. H. E. and Piche, R.: Stability of Follower-Force Rods with Weight, J. Eng. Mech., 110, 3 (1984), pp. 367-379.

13) Pradhan, S. and Datta, P. K.: Dynamic Instability Characteristics of a Free-Free Missile Structure under a Controlled Follower Force, Aircraft Eng. Aerospace Tech., 78, 6 (2006), pp. 509-514.

14) Irani, S. and Kavianipour, O.: Effect of a Flexible Joint on Instability of a Free-Free Joined Bipartite Beam under the Follower and Transversal Forces, J. Zhejiang University Science, 2009, Doi:10.1631/ jzus.A0820621

15) Bazoune, A. and Khulief, Y. A.: Shape Functions of Three Dimensional Timoshenko Beam Element, J. Sound Vib., 259 (2003), pp. 335-351. 